\title{
Hydrodynamic theory of plastic flows with conversion.
}

\author{
N. Kirova ${ }^{1}$ and S. Brazovskii ${ }^{2}$ \\ ${ }^{1}$ LPS, Université Paris-Sud, Orsay, France. \\ ${ }^{2}$ LPTMS, Université Paris-Sud, Orsay, France \\ http://ipnweb.in2p3.fr/ lptms/membres/brazov/
}

October 8, 2004

\begin{abstract}
Experimental studies show that the sliding state is essentially inhomogeneous with a variety of effects near contacts, in the bulk or at obstacles. We present a general scheme to approach the space time evolution of deformations, electric fields and currents with particular emphasize to the current conversion process in Charge Density Waves. We derive general equations for the multi-fluid hydrodynamics of plastic flows which allow, in principle, to study the transformation of injected carriers from normal electrons to new periods, collective motion in constrained geometry as well as the plastic contribution to non-linear current-voltage characteristics. [3]
\end{abstract}

\section{Introduction.}

Plastic deformations and flows are currently the focus of studies of Electronic Crystals (charge/spin density waves (CDW/SDW, DW) and interface $2 \mathrm{~d}$ Wigner crystals) and of vortex lattices. Thus, dislocations are supposed to participate in depinning, in phase slips, in narrow band noise generation, and also arise in contact structures. The point defects $( \pm 2 \pi$-solitons as add-atoms and vacancies or also as nucleus dislocation loops) compete with electrons as normal carriers. Also they provide a material for the climb of dislocations 
required for their expansion. Metastable plastic deformations are originated in the course of the DW sliding over the host lattice imperfections. Peculiarities of dislocations in DW crystals are related to their ultimate necessity to transfer a normal current into the coherent crystal flow, to an anomalous elastic theory due to long range Coulomb forces, to fast conversion of normal carriers into nucleus dislocations, to combined symmetry for the SDW case. Properties of dislocations are strongly affected by the Coulomb forces and hence depend on screening facilities of free carriers.

The coexistence and mutual conversion of electrons and intrinsic defects results in a nonlinear hydrodynamics for two fields: the DW phase and the electric potential, and for two fluids: electrons and point defects.

DW is a superstructure $\sim \cos \left[\vec{q}_{0} \vec{r}+\varphi\right]$ produced by modulations of electronic charges and atomic displacements or of electronic spins, for Chargeor Spin DWs correspondingly. With respect to deformations it is a kind of elastic uniaxial crystal, which is characterized by distortions of the DW phase $\varphi=\varphi(\vec{r})$. The DW sliding is ultimately related to a current conversion process and here we immediately face the problem of topological defects like solitons, phase slips, dislocation lines/loops. Their conversion passes through consecutive steps: free electrons injected from the contact transform dynamically to amplitude solitons. By kinetic processes of pair collisions the amplitude solitons undergo to $2 \pi$ phase solitons forming phase slips. Their subsequent aggregation forms edge dislocation lines (D-lines) or loops (Dloops) expanding across the sample. They are created near contacts, by surface strains, crystal shape steps and pinning centers.

The best introduction, with many illustrations, to dislocations in CDWs can be found in [1]. In relation to depinning the dislocations in CDW were discussed recently in [2].

\section{Kinematics of dislocations.}

Going around the D-line $\vec{\tau}$ or crossing the D-loop, the phase $\varphi(\vec{r})$ gains $2 \pi$ increments at some, largely arbitrary chosen, surface $\vec{P}$, see Fig.1, Fig.2 below. It makes the phase to be either a continuous multiple value function or, as another choice, a discontinuous and not uniquely defined function. The situation is further complicated if defects are in motion or evolution which makes these discontinuity time dependent. Now the phase can be characterized only by its derivatives, to be defined locally and instantaneous 
$\varphi_{i}:$

$\partial_{x} \varphi \rightarrow \varphi_{x}, \partial_{y} \varphi \rightarrow \varphi_{y} ; \partial_{z} \varphi \rightarrow \varphi_{z} ;\left(\varphi_{x}, \varphi_{y}, \varphi_{z}\right)=\left(\omega_{x}, \omega_{y}, \omega_{z}\right)=\vec{\omega} ; \partial_{t} \varphi \rightarrow \varphi_{t}$

The velocity $\varphi_{t}$, strains $\vec{\omega}$ and associated stresses $\vec{\sigma}$ are unique quantities having only physical singularities at D-lines themselves and they allow for averaging for their distributions and motion. But at presence of defects they are not derivatives of the same phase: the usual single equation for the elastic dissipative media multiplies to four equations for these for fields (in addition to equations for defects themselves, normal carries and electric field).

Furthermore, averaging over defects requires to carefully distinguish two types of their motion: glide and climb.

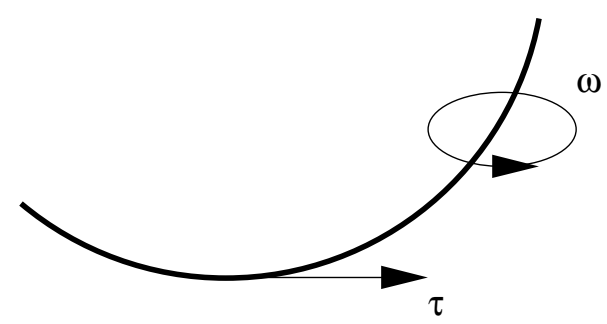

Figure 1: Dislocation line $\tau$ with circulations of the phase gradient $\omega$.

In the course of the glide, the D-loop does not cross chains; this is a conservative process which preserves the difference $n_{n}=n_{e}-n_{h}$ in concentrations of electrons $n_{e}$ and holes $n_{h}$ of normal carriers (the "amorphous component", add-atoms and vacancies in terms of conventional crystals). The glide is allowed only in the direction of the Burgers vector of these edge dislocations, that is along the chains axis $x$, hence the strictly uniaxial current of defects $\vec{j}_{d}=\left(j_{d}, 0,0\right)$, see Fig. 3 . 
In the course of the climb, the D-loop grows or shrinks changing the number of embraced chains, see fig.4. This change provides the definition for the density of defects $n_{d}$ which is the number of embraced chains (i.e. the normalized area of D-loops) per unit volume. The total speed $\dot{n}_{d}$ of the climb is uniquely related to the current conversion rate $R$ :

$$
\partial n_{d} / \partial t+\partial_{x} j_{d}=d n_{d} / d t=-d n_{n} / d t=R
$$

There is a substantial simplification for above problems which is not quite an expected result. There exists a self-averaging uniquely defined generalization of the phase, the function $\chi$ defined such that

$$
\frac{\partial \chi}{\partial t}=\left\langle\varphi_{t}\right\rangle+2 \pi j_{d} ; \frac{\partial \chi}{\partial x}=\left\langle\omega_{x}\right\rangle-2 \pi n_{d}, \frac{\partial \chi}{\partial y}=\left\langle\omega_{y}\right\rangle, \frac{\partial \chi}{\partial z}=\left\langle\omega_{z}\right\rangle
$$

The observation allows to bring multiple variables and their equations to the single one as we shall demonstrate below.

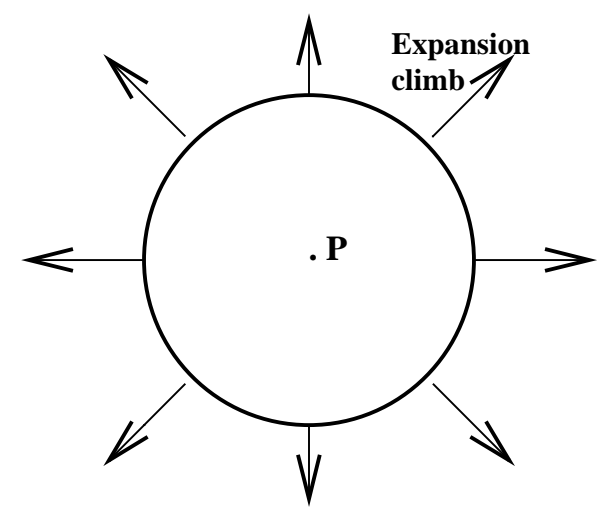

Figure 3: Expansion of the dislocation loop in the course of its climb. Arrows show the climb direction. The surface vector $\vec{P}$ is perpendicular to the plane.

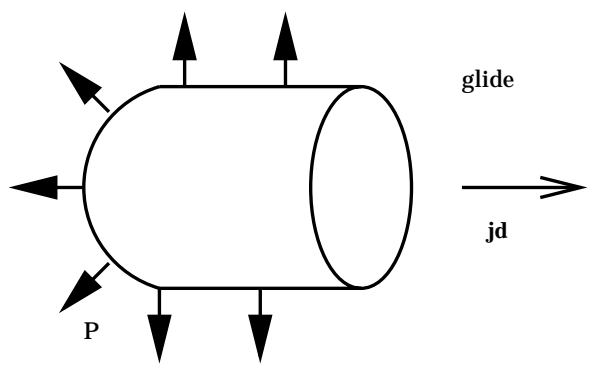

Figure 4: Propagation of the dislocation loop in the course of its glide toward the defect current $\vec{j}_{d}$ direction. Vectors show the surface of the phase discontinuities. 


\section{Material relations and final results}

In applications, we shall keep in mind the specific for CDWs functional of the free energy $W$ (density per chain area $s)$ :

$$
\begin{array}{r}
W=\frac{\hbar v_{F}}{4 \pi}\left[\omega_{x}^{2}+\alpha_{y} \omega_{y}^{2}+\alpha_{z} \omega_{z}^{2}\right]+\frac{e}{\pi} \omega_{x} \Phi-\frac{\varepsilon_{h} s}{8 \pi}(\nabla \Phi)^{2} \\
e \Phi\left(n_{e}+n_{0}\right)+\left(e \Phi+\frac{1}{2} \hbar v_{F} \omega_{x}\right) n_{i}+W_{l o c}\left(n_{n}, n_{d}\right)
\end{array}
$$

Here $\alpha_{x, y}$ are the share modules, $\varepsilon_{h}$ is the host dielectric constant, $\Phi$ is the electric potential interacting with charges of the DW $e \omega_{x} / \pi$ and of normal carries in the material $n_{n}=n_{e}+n_{i}$ and in the contact metal $n_{0} . W_{l o c}\left(n_{n}, n_{d}\right)$ is the free energy of normal carriers at their local equilibrium. Here we distinguish two types of free carriers [7]:

- Intrinsic, their spectrum is formed by the DW gap and their energy follows the Fermi level breathing $\delta E_{F}=\hbar v_{F} \omega_{x} / 2$.

- Extrinsic, they belong to other bands or to another well decoupled DW (this is the case of the $\mathrm{NbSe}_{3}$ between the two transitions).

Below we shall normalize, without changing notations, all potentials to $e N_{F}$, all energies to the metallic density of states $N_{F}=2 /\left(\pi \hbar v_{F}\right)$ so that they are measured in inverse lengths (and forces in inverse square lengths). We shall introduce also the Debye screening length of the parent metal $r_{0}=\kappa^{-1}$, $\kappa^{2}=8 e^{2} / s \hbar v_{F}$.

The local balance of forces $\mathcal{F}$ and stresses $\vec{\sigma}$ for the viscous media reads

$$
\begin{aligned}
\vec{\nabla} \vec{\sigma} & =\mathcal{F}_{f r c}=\mathcal{F}_{p i n}+\frac{\gamma}{\pi} \partial_{t} \varphi ; \vec{\sigma}=\pi \frac{\delta W}{\delta \vec{\omega}} \\
\sigma_{x} & =\frac{\omega_{x}}{\pi}+\Phi+n_{i}, \sigma_{y}=\frac{\alpha_{y} \omega_{y}}{\pi}, \sigma_{z}=\frac{\alpha_{z} \omega_{z}}{\pi}
\end{aligned}
$$

Here $\mathcal{F}_{f r c}$ is the friction force by the host which is usually taken as the viscous force $\sim \partial_{t} \varphi$ generalizable to a non-linear law of $\mathcal{F}_{\text {pin }}$. We have suggested for simplicity a linear dependence of the friction force $\mathcal{F}_{f r c}\left(\partial_{t} \varphi\right)$ (e.g. a normal viscosity at high velocities) plus the remnant pinning force $\mathcal{F}_{\text {pin }}$. We need 
also two other potentials $U$ and $V$ which play a principle role in behavior of carriers and defects,:

$$
U=\Phi+n_{i}+\frac{1}{\pi} \omega_{x}, V=\frac{\delta W}{\delta n_{i}}=\Phi+\frac{1}{\pi} \omega_{x}
$$

The potential $U=\sigma_{x}$ is the longitudinal stress. $2 U$ is the energy paid to distort the DW elastically by one period, that is $U$ refers to one electron in the ground state. $V$ is the potential experienced by intrinsic free carriers. The most efficient equations can be written for the pair of variables $\chi$ and $\Phi:$

$$
\begin{aligned}
\frac{1}{\pi}\left(\hat{\Delta}-\gamma \partial_{t}\right) \chi & =-\frac{\partial}{\partial x} \Phi-2 \gamma j_{d}-\frac{\partial}{\partial x}\left(n_{i}+2 n_{d}\right) \\
r_{0}^{2} \Delta \Phi+\frac{1}{\pi} \partial_{x} \chi & =-\left(n_{0}+n_{i}+n_{e}+2 n_{d}\right) \quad r_{0}^{-2}=\frac{8 e^{2}}{\hbar v_{F} \varepsilon_{h} s}
\end{aligned}
$$

The last one is the Poisson equation obtained by variation $W$ over $\Phi$. The equations (6) and (7) must be supplemented by the conservation law for the total charge

$$
\frac{d}{d t}\left(n_{i}+n_{e}+n_{0}+2 n_{d}\right)=0, \frac{d n_{a}}{d t}=\frac{\partial n_{a}}{\partial t}+\vec{\nabla} \vec{j}_{a} ; a=0, e, i, d ; \vec{j}_{d}=\vec{\nu} j_{d}
$$

For all normal carries, $a \neq d$, the currents $\vec{j}_{a}$ are given by drifts with mobilities $G_{a}$ in the combined gradient of the appropriate potential $V_{a}$ and the local chemical potential dependent on their concentration:

$\vec{j}_{a}=-G_{a} \nabla \mu_{a} ; \mu_{a}=\frac{\delta W}{\delta n_{a}}=V_{a}+\mu_{l o c}^{a}, \quad \mu_{a}^{l o c}=\frac{\delta W_{l o c}}{\delta n_{a}} ; V_{0}=V_{1}=\Phi, V_{i}=V$

At first sight, for defect $V_{d}$ might be their energy per chain $2 U$ which works indeed to determine there thermodynamic balance. But it would be a mistake to use $-2 U^{\prime}$ as the driving force like for other potentials in (9). The glide of defects is enforced by share strains, the perpendicular gradients

$$
\begin{aligned}
-\partial_{x} V_{d} & =2 \hat{\Delta}_{\perp} \chi \neq-2 \partial_{x} U \\
\hat{\Delta}_{\perp} \chi+\partial_{x} U & =\gamma \partial_{t} \chi-2 \pi \gamma j_{d} \neq 0
\end{aligned}
$$

If chains are not packed particularly distantly (which may be the case of artificial Wigner crystals of e.g. systems of quantum wires) $r_{0}$ is the smallest, 
atomic, length scale. In this case the relations between $\Phi, U, V \chi$ and $n_{a}$ can be simplified as:

$$
U=V+n_{i}=\Phi-n_{0}-n_{e} ; \pi^{-1} \partial_{x} \chi=-2 n_{d}-n_{0}-n_{i}-n_{e}
$$

Now the closed equation can be written for the phase alone:

$$
\frac{1}{\pi}\left[r_{0}^{2} \Delta_{\perp}\left(\Delta_{\perp}-\gamma \partial_{t}\right)-\partial_{x}^{2}\right] \chi=\partial_{x}\left[n_{n}+2 n_{d}\right]
$$

which high order of non-locality is related to the Coulomb hardening. We see that the driving force is provided only by the parallel gradient of the total concentration of normal carriers and defects, nothing else.

Averaging over the sample cross-section \langle\rangle$_{r_{\perp}}$ we arrive at the most simple set of relations

$$
\begin{gathered}
\frac{1}{\pi} \frac{\partial \chi}{\partial x}+n_{0}+n_{e}+n_{i}+2 n_{d}=0 \\
-\frac{1}{\pi} \frac{\partial \chi}{\partial t}+j_{n}+2 j_{d}=J=\text { cnst }
\end{gathered}
$$

The Eqs. (13) - (14) are nothing but conservation laws for the charge and the current, which became local in this case. The total current cannot depend on $x: J=J(t)$ and the total charge is not perturbed as the first of above equations reads. The equation (14) can be also written as

$$
U_{x}^{\prime} G_{c}+\mu_{n}^{\prime} G_{n}=-J(t) ; \quad G_{n}=G_{i}+G_{e} ; \quad G_{c}=\frac{1}{\gamma}
$$

which is the Kirchoff law for parallel circuits of collective and normal conductions. For this limit we can also write relations between $\chi$ and potentials:

$$
\frac{\partial \Phi}{\partial x}-\frac{\gamma}{\pi} \frac{\partial}{\partial t} \chi=F_{p i n}-2 \gamma j_{d}+\frac{\partial}{\partial x}\left(n_{0}+n_{e}\right) \frac{\partial U}{\partial x}-\frac{\gamma}{\pi} \frac{\partial}{\partial t} \chi=\mathcal{F}_{p i n}-2 \gamma j_{d}
$$

The Eq. (??) can be written as the electric field actions

$$
E=-\gamma \partial_{t} \chi+2 \gamma j_{d}+\partial_{x} n_{n}
$$

It helps to resolve the dilemma of the basic viscoelastic equation

$$
E=\gamma \dot{\varphi}+k \varphi^{\prime \prime}:
$$


how the electric field $E$ is partitioned between the frictional $\gamma \dot{\varphi}$ and elastic $k \varphi^{\prime \prime}$ resistances. We see that strictly speaking $E$ drives the DW motion $\dot{\chi}$, the current of defects $j_{d}$ (together $=$ total collective current) and also $E$ withstands the gradient of normal carriers concentration which hiddenly incorporates the elastic force.

\section{Conclusions.}

The presented results provide several phenomenologically rigorous relations between observable quantities. They allow for some, not quite expected, interpretations particularly on the control variables and driving forces. They can provide a basis for analysis of even most complicated experimental situation at the level which was earlier well recognized in theory and practice of semi- and superconductors.

Acknowledgments: Authors acknowledge the support from the INTAS grant 2212 .

\section{References}

[1] D. Feinberg, J. Friedel, "Imperfections of charge density wave in blue bronzes", in "Low-Dimensional Electronic Properties of Molybdenum Bronzes and Oxides, Edited by C. Schlenker (Kluwer Academic Publishers,Dordrecht, 1989) p. 407-448.

[2] S.Brazovskii, T.Nattermann, "Pinning and Sliding of Driven Elastic Systems: from Domain Walls to Charge Density Waves", Advances in Physics, 53 (2004) 177-253 (cond mat/0312375).

[3] N.Kirova, S.Brazovskii, J. Physique IV, 12 (2002) Pr9-173 (Proceedings of ECRYS-2002).

[4] S.Brazovskii, N.Kirova, "Electron Selflocalization and superstructures in quasi one-dimensional dielectrics" Soviet Scientific Reviews, Sec. A, Phys. Rev., I. M. Khalatnikov Ed., v. 5, 99 (Harwood Acad. Publ.,1984)

[5] S.Brazovskii, "Solitons in charge density wave crystals", in Modern Problems in Condensed Matter Science, v. 25, 
Charge density waves in solids, L.Gor'kov, G.Grüner Eds.

(Elsevier Sci. Publ., Amsterdam, 1990), p. 425.

[6] S.Brazovskii, S.Matveenko, J. Physique I, 2 (1992) 725.

[7] S.Brazovskii, N.Kirova, H.Requardt, F.Ya. Nad, P.Monceau, R.Currat, J.E.Lorenzo, G.Grübel, Ch.Vettier, Phys. Rev. B 61 (2000) 10640. 